Science, Technology and Development 35 (1): 42-46, 2016

ISSN 0254-6418 / DOI: 10.3923/std.2016.42.46

(C) 2016 Pakistan Council for Science and Technology

\title{
Monitoring Molecular Heterogeneity of $\beta$-thalassemia Syndrome in District Nowshehra
}

\author{
${ }^{1}$ Muhammad Shakeel, ${ }^{1}$ Muhammad Ishfaq, ${ }^{3}$ Shoaib Ur Rehman, \\ ${ }^{2}$ Tabassum Yaseen and ${ }^{4}$ Muhammad Arif Lodhi \\ ${ }^{1}$ Department of Biotechnology, Bacha Khan University, Charsadda, Khyber Pakhtunkhwa, Pakistan \\ ${ }^{2}$ Department of Botany, Bacha Khan University, Charsadda, Khyber Pakhtunkhwa, Pakistan \\ ${ }^{3}$ Department of Biotechnology, Bannu University of Science and Technology, \\ Bannu, Khyber Pakhtunkhwa, Pakistan \\ ${ }^{4}$ Department of Biochemistry, Abdul Wali Khan University, Mardan, Khyber Pakhtunkhwa, Pakistan
}

\begin{abstract}
Among the reported single gene disorders/syndrome, hemoglobin infection is the most common and widespread genetic disorder. Thalassemia arises from defect in red blood cell proteinaceous component (hemoglobin), which carries oxygen and may lead to anemia. Present study aimed to detect the common molecular abnormalities of $\beta$-thalassemia syndrome in district Nowshehra, Khyber Pakhtunkhwa, Pakistan. This work was conducted at Abdul Wali Khan University, Mardan, the reports collecting blood samples from patients (average age 13 years) and their families with $\beta$-thalassemia major $(\mathrm{n}=13$ families) belonged to district Nowshehra. The assembled blood samples from the patients were tested for the presence of most common mutations, using Polymerase Chain Reaction (PCR). This study reports six known mutations (IVS-1-5, FSC 8/9, CD 41/42, IVS-1-1, CD 15 and FSC-5) comprising 90\% of the total $\beta$-thalassemia genes in Pakistan. In our study, IVS 1-5 and FSC-8/9 were the most widespread $\beta$-thalassemia gene mutation detected in patients belonging to district Nowshehra. The findings of the study can be used to get an idea about the most common mutation in this region and in designing pre-natal programmes to control the genetic disease.
\end{abstract}

Key words: $\beta$-thalassemia, common mutations, pre-natal diagnosis

\section{INTRODUCTION}

Thalassemia demonstrates inherited diseases of hemoglobin which has been reported for the first time from the developed and highly advanced countries like the United States. The word "Thalassemia" has been derived from Greek, which means "Sea blood". In the past, people believed that this fatal disease exists in Mediterranean regions only but, later on, it was shown by a number of studies that the disease may be found in all parts of the world (Williams et al., 2005; Kremastinos, 2007; Kremastinos et al., 2010). Recent studies have stated that the occurrence of thalassemia is rare in developed countries due to excellent health-related facilities and regular screening of pregnant women for the presence of the mentioned disorder.

According to several reports, thalassemia is the first genetic disease which has been studied on molecular level and resulted in enough knowledge about its common mutations and occurrence (Joly et al., 2009; Sankaran et al., 2011). This has increased our knowledge about its forms, symptoms and other characteristics, which can be used in devising strategies for its control in our population. Educating general public about its occurrence, screening of pregnant women, improving the socioeconomic conditions and motivating people to discourage close-marriages can prove helpful in controlling thalassemia.

It is caused as a result of heritable mutation in globin chains of hemoglobin (Watterson, 1975; Rund and Rachmilewitz, 2005). Different forms of hemoglobins can be found at different developmental stages in humans such as $\mathrm{HbA}$ (known as major hemoglobin) in normal human. It is called major hemoglobin as it comprises $90 \%$ of the total hemoglobin in the body. The HbA2 or minor hemoglobin can be found in normal adults comprising $2-3 \%$ of the total hemoglobin. The HbF has been reported to be the major hemoglobin in infants (Badens et al., 2000) but it is found in small proportion in adults.

In adults and infants, hemoglobin has chains associated with beta, delta or gamma chains. At embryo stage, the Z-chains can be observed easily (Wood et al., 2005). Two different forms of thalassemia (a, b) can arise, when any mutation occurs in these chains during synthesis process. Mutation can also occur during synthesis of other chains (delta, beta and gamma) called

Corresponding Author: Muhammad Shakeel, Department of Biotechnology, Bacha Khan University, Charsadda, Khyber Pakhtunkhwa, Pakistan 
as $\delta \beta$ or $\varepsilon \gamma \delta \beta$ thalassemia. It does not follow the Mendalian segregation fashion for recessive genes.

Thalassemia major refers to more severe defect which can be found in homozygous form. The situation in which one of the defective/mutated globin gene is inherited is called "Carrier". The objective of the current study was to look at the common molecular anomalies of $\beta$-thalassemia disease in District Nowshehra, Khyber Pakhtunkhwa which could provide useful information for establishing thalassemia diagnosis and parental screening Centre.

\section{MATERIALS AND METHODS}

The study was conducted at the Abdul Wali Khan University, Mardan. The blood samples from 400 patients and their families were taken in ethylene-diamine-tetraacetic acid (EDTA) solution. The patients involved in the present study were on blood transfusion and diagnosed as $\beta$-thalassemia major patients by earlier studies, using basic hematological parameters, peripheral blood morphology and hemoglobin electrophoresis procedures. About 400 patients with $\beta$-thalassemia major disease aged 13 years were studied. Among the studied patients, 250 were male and 150 were female. The collected blood samples were tested for the presence of common mutations taking the help of modified amplification of refractory mutation scheme (ARMS). In this study, $63 \beta$-thalassemia chromosomes were analyzed to determine their mutation frequency in the residents of district Nowshehra. To screen known $\beta$-thalassemia causative mutation, PCR based tests were performed.

The study was conducted with five mutant primers (IVSI-1 $(\mathrm{G} \rightarrow \mathrm{T})$, IVSI-5 $(\mathrm{G} \rightarrow \mathrm{C})$, Del 619, Fr 41-42 (-TTCT) and Fr 8-9 (+G)). Gentra Pure Gene kit (Minneapolis, Minnesota, USA) was used for extraction of DNA from the collected blood samples according to supplier's instructions. Amplification of refractory mutation scheme was the tool used for screening of common mutations. In PCR, two tubes for each sample were amplified, one tube for normal gene primers and the other one for mutant gene primers. Primers A and B were used as control primers to ensure that there is no problem with PCR conditions.

The PCR was executed in final volume of $20 \mu \mathrm{L}$ reaction $(0.5 \mu$ g template DNA, 10 picomolar of each of the primers, 2.5 unit Taq DNA polymerase, $0.2 \mathrm{mM}$ of each of the deoxynucleotide-tri-phosphate (dNTP) in a solution consisting of $10 \mathrm{mM}$ tris- $\mathrm{HC} 1,50 \mathrm{mM} \mathrm{KCl}$ and $1.2 \mathrm{mM} \mathrm{MgCl} 2$ ). The thermal cycler was set for 25 cycles as: Denaturation at $94^{\circ} \mathrm{C}$ for $1 \mathrm{~min}$, primer annealing at $65^{\circ} \mathrm{C}$ for $1 \mathrm{~min}$, extension at $72^{\circ} \mathrm{C}$ for $1.5 \mathrm{~min}$ and final extension at $72^{\circ} \mathrm{C}$ for $10 \mathrm{~min}$. About $10 \mu \mathrm{L}$ of PCR product mixed with $3 \mu \mathrm{L}$ of a loading buffer was run on $2 \%$ agarose gel stained with ethidium bromide. For electrophoresis, $100 \mathrm{~V}$ for $60 \mathrm{~min}$ was used for separation of amplified PCR product. Either 50 or 100 bp DNA as size ladders were used. The PCR product was visualized under ultraviolet light.

\section{RESULTS AND DISCUSSION}

Blood samples collected from 13 transfusion dependent $\beta$-thalassemia patients and their family members provided useful data. Among the six $\beta$-thalassemia mutations identified in Pakistan, two $\beta$ mutations, i.e., IVS-1-5 and FSC-8/9 (Fig. 1 and 2) were identified in the current study, using PCR approach.

Some samples were noted to have been amplified with mutant primers, they were likely homozygous for the tested mutation. Samples amplified with normal primers were presumably negative for the tested mutation. Samples, which amplify with both normal and mutant primers, are believed to be heterozygous (carrier) for that mutation.

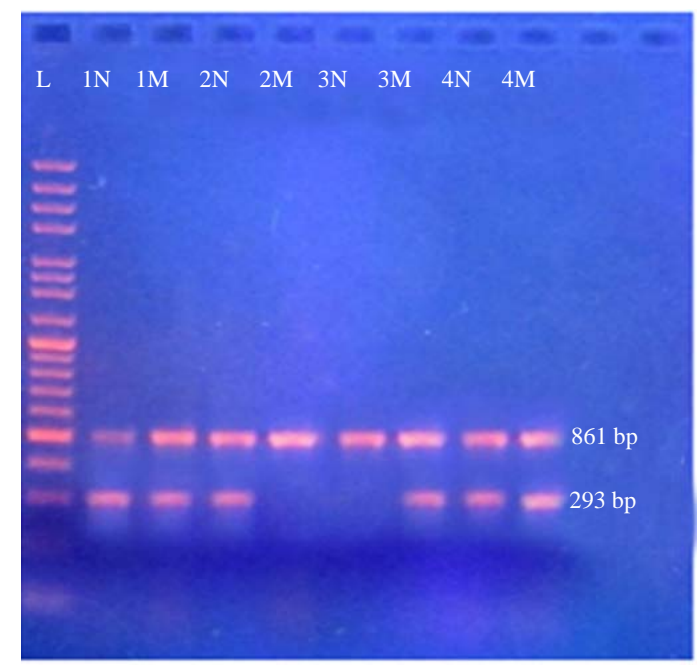

Fig. 1: PCR amplified product of IVS-1-5 mutation gene. Primers producing 861 bp fragments was used as control primer, common C (forward primer) and IVS-1-5 Mt (mutant) or N (normal), used as reverse primer amplifies PCR product of $293 \mathrm{bp}$. M: Mutant products, N: Normal PCR products, L: $1 \mathrm{~kb}$ DNA ladder. The father DNA is run in lane $1 \mathrm{~N}$ and $1 \mathrm{M}$, it represents that father is heterozygous. The mother DNA is run in lane $2 \mathrm{~N}$ and $2 \mathrm{M}$, it represents that mother is negative for this mutation. The 1st child DNA is run in lane $3 \mathrm{~N}$ and $3 \mathrm{M}$, it represents that the 1st child is homozygous. The 2nd child's DNA is run in lane $4 \mathrm{~N}$ and $4 \mathrm{M}$, it represents that he is heterozygous 


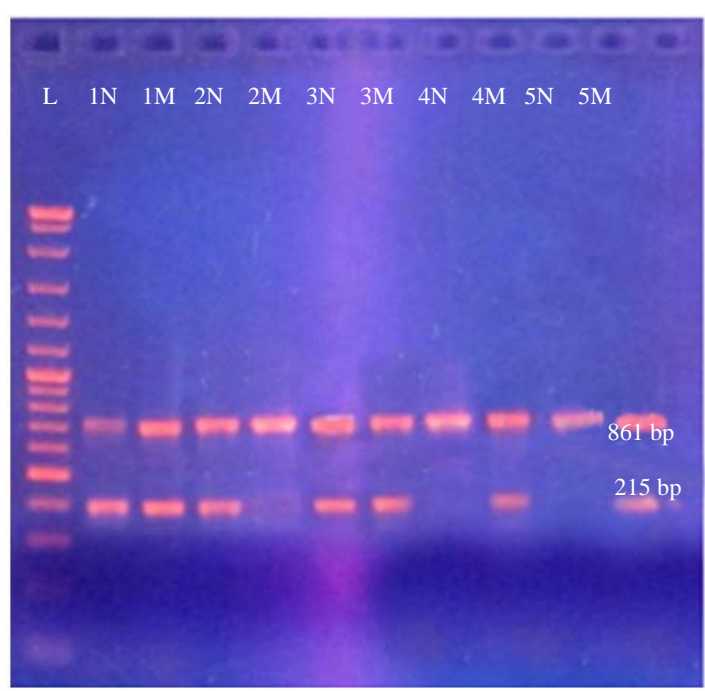

Fig. 2: PCR amplified product of FSC 8-9 mutation gene. Control primers producing 861 bp fragments used as control, common $\mathrm{C}$ (forward primer) and FSC 8-9 Mt (mutant) or N (normal), used as reverse primer amplifies PCR product of 293 bp. M: Mutant products, N: Normal PCR products, L: $1 \mathrm{~kb}$ DNA ladder. The father DNA is run in lane $1 \mathrm{~N}$ and $1 \mathrm{M}$, it represents that father is heterozygous. The mother DNA is run in lane $2 \mathrm{~N}$ and $2 \mathrm{M}$ it represents that mother is negative for this mutation. The 1st child DNA is run in lane $3 \mathrm{~N}$ and $3 \mathrm{M}$, it represents that the 1st child is heterozygous. The 2nd child DNA is run in lane $4 \mathrm{~N}$ and $4 \mathrm{M}$, it represents that 2nd child is homozygous. The 3rd child DNA is run in lane $5 \mathrm{~N}$ and $5 \mathrm{M}$, it represents that 3rd child is homozygous

In PCR, addition of control primers helped in predicting the expected size of amplicon and best interpretation of the results in the study. Among the studied 63 chromosomes, molecular defects were found in $13 \beta$-thalassemia chromosomes. The $\beta$-thalassemia molecular defect was detected in 13 patients as homozygous and in the 26 patients as heterozygous (carrier). Our results of getting two common mutations are in agreement with the results of Ahmed et al. (2002). This study reinforces that IVS-1-5 and FSC 8/9 are the most common $\beta$-globin gene mutation that underlie $\beta$-thalassemia in Pakistan.

The high frequency of $\beta$-thalassemia in Pakistani inhabitants is due to several factors, including marriages between close relatives, exposure of humans to mutagens and increased birth rate. High frequency of $\beta$-thalassemia is one of the reasons for declaring Pakistan as a country with the highest transfusion dependent children
(Majeed et al., 2013; Malik et al., 2009; Alwan et al., 1997). According to WHO (2001) reported that the allele frequency in Pakistan was about $5 \%$ and the percentage of individual's carriers in couples was $25 \%$. About 400 $\beta$-thalassemia mutations or molecular defects have been detected worldwide only $19 \beta$-thalassemia disorders have been revealed in Pakistan (Khan et al., 1995). As a complete treatment of the disease has not been discovered as yet, the available treatment strategies include iron chelation remedy, transfusion of blood and bone marrow transfer. Iron chelation remedy and transfer of thalassemia free blood to all patients is difficult due to its high cost and reduced medical facilities (Ahmed et al., 2002). High treatment cost is one of the reasons for not being able to control this disorder in our country. Significant success has been achieved in controlling the spread of $\beta$-thalassemia in developed countries by controlling the birth rate from the infected or carrier parents. In these countries, pre-marital screening (Saffi and Howard, 2015) and screening of pregnant women are regularly done for the presence of signs of $\beta$-thalassemia (Tuzmen et al., 1996; Cousens et al., 2010; Cao, 2002). If any symptoms, relating to this disease, is observed in pregnant women, detailed investigation of the both women and their husbands and/or their parents is done and then it is decided whether the fetus is worth abortion or could be retained without getting effects of thalassemia.

However, such practices cannot be done in developing countries, like Pakistan, due to limited allocated funds towards health and large number of population, i.e., more than 150 million population. In addition, lack of awareness is another persistent problem which has resulted in increased number of thalassemia cases in Pakistan. As a result, most of the children are born with this disease which reduces the chances to control it before birth (Baig et al., 2005). A new strategy developed for proper screening of the infected or doubtful families include index case or retrospective inductive screening (Old, 2003), which is economical, feasible and more practicable than other screening methods in Pakistan. In the current study, the representative population was screened from district Nowshehra. This study reports six $\beta$-thalassemia causative mutations (i.e., IVS-1-5, FSC 8/9, CD 41/42, IVS-1-1, CD 15, FSC-5) and deletion of 619 bp mutation in $\beta$ globin gene identified by PCR.

In our study, out of 13 unrelated transfusion dependent patients, 7 carried IVS-1-5 and 6 carried FSC 8/9 mutation. While analyzing two extended families, each with an index case of $\beta$-thalassemia, $66.7 \%$ were detected as carriers, having $33 \%$ risk of producing an affected child if married to a carrier. These results show 
higher carrier frequency (66.7\%) as compared to previous (31\%) reports (Ahmed et al., 2002).

It is worth mentioning that the common mutations, detected by our study, are a subset of total mutations identified in $\beta$-globin gene worldwide. This study illustrated that $90 \%$ of mutations causing $\beta$-thalassemia in Nowshehra regions has been evolved from seven mutations. This study intensifies the need for conducting index case screening of large families rather than general population screening to prevent the spread of $\beta$-thalassemia in developing countries, like, Pakistan.

\section{CONCLUSION}

The $\beta$-thalassemia arises from defect in red blood cell proteinaceous component (hemoglobin), which carries oxygen and that may lead to anemia. It is the most prevalent genetic disease in developing countries, like Pakistan, due to lack of social awareness, appropriate knowledge and enough medical facilities. Its occurrence has been reduced to a large extent in developed countries due to a regular pre-marital screening of couples and screening of pregnant women for the presence of any signs of this genetic disorder. Among six different $\beta$-thalassemia mutations identified in Pakistan so far, two $\beta$-thalassemia mutations, i.e., IVS-1-5 and FSC-8/9 were identified by the present study, using PCR approach. These two mutations were found to be the most common $\beta$-thalassemia mutation in patients belonging to District Noshehra.

This study recommends index case or retrospective inductive screening in Pakistan despite general population based screening as it is economical and feasible. This study also emphasizes on establishing thalassemia screening centre and a regular monitoring of pregnant women in rural areas of Pakistan, which could be helpful in reducing the incidence of this genetic disease.

\section{REFERENCES}

Ahmed, S., M. Saleem, B. Modell and M. Petrou, 2002. Screening extended families for genetic hemoglobin disorders in Pakistan. New Engl. J. Med., 347: 1162-1168.

Alwan, A., B. Modell, A. Bittles, A. Czeilel and H. Hamamy, 1997. Community control of genetic and congenital disorders. WHO EMRO Technical Publications Series No. 24. World Health Organization, Office for the Eastern Mediterranean.

Badens, C., I. Thuret and D. Lena-Russo, 2000. Les syndromes thalassemiques. Revue Francaise des Lab., pp: 23-27.
Baig, S.M., F. Rabbi, U. Hameed, J.A. Qureshi and Z. Mahmood et al., 2005. Molecular characterization of mutations causing $\beta$-thalassemia in Faisalabad Pakistan using the amplification refractory mutation system (ARMS-PCR). Indian J. Hum. Genet., 11: 80-83.

Cao, A., 2002. Carrier screening and genetic counselling in $\beta$-thalassemia. Int. J. Hematol., 2: 105-113.

Cousens, N.E., C.L. Gaff, S.A. Metcalfe and M.B. Delatycki, 2010. Carrier screening for $\beta$-thalassaemia: A review of international practice. Eur. J. Hum. Genet., 18: 1077-1083.

Joly, P., P. Lacan, C. Garcia, N. Couprie and A. Francina, 2009. Identification and molecular characterization of four new large deletions in the $\beta$-globin gene cluster. Blood Cells Mol. Dis., 43: 53-57.

Khan, S.N., A.U. Zafar and S. Riazuddin, 1995. Molecular genetic diagnosis of beta thalassemia in Pakistan. J. Pak. Med. Assoc., 45: 66-70.

Kremastinos, D.T., 2007. Heart failure in $\beta$-thalassaemia: A local or universal health problem? Hellenic J. Cardiol., 48: 189-190.

Kremastinos, D.T., D. Farmakis, A. Aessopos, G. Hahalis, E. Hamodraka, D. Tsiapras and A. Keren, 2010. $\beta$-thalassemia cardiomyopathy history, present considerations and future perspectives. Circ.: Heart Failure, 3: 451-458.

Majeed, T., M.A. Akhter, U. Nayyar, M.S. Riaz and J. Mannan, 2013. Frequency of $\beta$-thalassemia trait families of thalassemia major patients, Lahore. J. Ayub Med. Coll. Abbotabad, 25: 58-60.

Malik, S., S. Syed and N. Ahmed, 2009. Complications in transfusion-dependent patients of $\beta$-thalassemia major. Pak. J. Med. Sci., 25: 678-682.

Old, J.M., 2003. Screening and genetic diagnosis of haemoglobin disorders. Blood Rev., 17: 43-53.

Rund, D. and E. Rachmilewitz, 2005. $\beta$-thalassemia. N. Engl. J. Med., 353: 1135-1146.

Saffi, M. and N. Howard, 2015. Exploring the effectiveness of mandatory premarital screening and genetic counselling programmes for $\beta$-Thalassaemia in the middle east: A scoping review. Public Health Genomics, 18: 193-203.

Sankaran, V.G., J. Xu, R. Byron, H.A. Greisman and C. Fisher et al., 2011. A functional element necessary for fetal hemoglobin silencing. New Engl. J. Med., 365: 807-814.

Tuzmen, S., G.O. Tadmouri, A. Ozer, S.M. Baig, H. Ozcelik, S. Basaran and A.N. Basak, 1996. Prenatal diagnosis of $\beta$-thalassaemia and sickle cell Anaemia in Turkey. Prenatal Diagnosis, 16: 252-258. 
WHO., 2001. Updated estimates of the frequency of the haemoglobin disorders in each country. World Health Organization (WHO). http://www.who.int/ncd/hgn/ haemogl.htm.

Watterson, G.A., 1975. On the number of segregating sites in genetical models without recombination. Theor. Popul. Biol., 7: 256-276.

Williams, T.N., T.W. Mwangi, S. Wambua, T.E.A. Peto and D.J. Weatherall et al., 2005. Negative epistasis between the malaria-protective effects of $\alpha^{+}$-thalassemia and the sickle cell trait. Nat. Genet., 37: 1253-1257.
Wood, E.T., D.A. Stover, M. Slatkin, M.W. Nachman and M.F. Hammer, 2005. The $\beta$-globin recombinational hotspot reduces the effects of strong selection around $\mathrm{HbC}$, a recently arisen mutation providing resistance to malaria. Am. J. Hum. Genet., 77: 637-642. 